UDC 517.97

M. T. Karaev (Inst. Math. and Mech. Azerbaijan Nat. Acad. Sci., Baku, Azerbaijan)

\title{
ON ESTIMATE OF NUMERICAL RADIUS \\ OF SOME CONTRACTIONS
}

\section{ПРО ОЦІНКУ ЧИСЛОВОГО РАДІУСА ДЕЯКИХ СТИСНЕНЬ}

For a numerical radius of an arbitrary nilpotent operator $T$ on a Hilbert space $H$, Haagerup and de la Harpe proved the inequality $w(T) \leq\|T\| \cos \frac{\pi}{n+1}$, where $n \geq 2$ is the nilpotency order of the operator $T$. In the present paper, we prove a Haagerup - de la Harpe-type inequality for a numerical radius of contractions from more general classes.

Хаагерун и Харп для числового радіуса довільного нільпотентного оператора $T$ у гільбертовому просторі $H$ довели нерівність $w(T) \leq\|T\| \cos \frac{\pi}{n+1}$, де $n \geq 2$ - порядок нільпотентності оператора $T$. У даній статті доведено нерівність типу нерівності Хаагеруна-Харпа для числового радіуса стиснень із більш загальних класів.

1. Introduction. Let $B(H)$ be an algebra of bounded linear operators acting on a complex Hilbert space $H$. The numerical range of the operator $T \in B(H)$ is called the set

$$
W(T)=\left\{(T x, x): x \in(H)_{1}\right\},
$$

where $(H)_{1}=\{x \in H:\|x\|=1\}$ is a unit sphere in the space $H$, and the numerical radius of an operator $T$ is defined by equality

$$
w(T)=\sup \{|\lambda|: \lambda \in W(T)\} .
$$

It is known that

$$
\frac{\|T\|}{2} \leq w(T) \leq\|T\|
$$

for any $T \in B(H)$ (see, for instance, [1, 2]). For concrete operators, obtaining for their numerical radius more subtle estimates than (1) is of special interest. So, in paper [3] Haagerup and de la Harpe proved the inequality

$$
w(T) \leq\|T\| \cos \frac{\pi}{n+1}
$$

for the numerical radius of an arbitrary nilpotent operator $T \in B(H)$ with the power of nilpotency $n \geq 2$ (i.e., $T^{n}=0$, but $T^{n-1} \neq 0$ ). In what follows other proofs of inequality (2) of Haagerup and de la Harpe are given in the works [4-8] (in [7], an evident description of the numerical range of any quadric operator on a Hilbert space is also given).

In the present paper, we prove the inequalities of the type of inequality (2) of Haagerup and de la Harpe for the numerical radius of contractions from the more general classes.

2. Notations and preliminaries. The notations in the paper are more or less standard. $C_{.0}$ denotes the class of all contractions $T$ on $H$, for which $\lim _{n} T^{*^{n}} x=0$ for all $x \in H$. $C_{00}$ is the class of all contractions $T$ on $H$, for which $\lim _{n} T^{n} x=\lim _{n} T^{*^{n}} x=0$ for all $x \in H$. It is clear that $C_{00} \subset C_{.0}$. It is well known (see, for instance, $[9,10]$ ) that if $T \in C_{.0}$, then it is unitary equivalent to the model operator $M_{\Theta}, M_{\Theta} f=P_{\Theta} z f, f \in K_{\Theta}$, where $P_{\Theta}$ is the orthogonal projection onto $K_{\Theta}$,

$$
K_{\Theta}=H^{2}(E) \ominus \Theta H^{2}\left(E^{\prime}\right)
$$


where $E=\operatorname{clos}\left(I-T T^{*}\right) H, E^{\prime}$ is a subspace of $E, H^{2}(E)$ is the Hardy space of $E$ valued analytic functions on the unit circle $\mathbb{D}=\{z \in \mathbb{C}:|z|<1\}$, and $\Theta$ is $B\left(E^{\prime}, E\right)$ valued bounded analytic function on $\mathbb{D}$ (the characteristic function of a contraction $T$ ) for which $\Theta(\xi) \Theta(\xi)^{*}=I_{E}$ for almost all $\xi \in \mathbb{T}=\partial \mathbb{D}$, i.e., $\Theta$ is a $*$-inner function (see [9]). In particular, if $T \in C_{00}$, then its characteristic function $\Theta \in B(E)$ is a two-sided inner function, that is $\Theta(\xi)^{*} \Theta(\xi)=\Theta(\xi) \Theta(\xi)^{*}=I_{E}$ for almost all $\xi \in \mathbb{T}$.

The proof of the following key lemma is contained, for instance, in [11].

Lemma 1. Let $k \geq 2$ be an integer. Then

$$
\max \left\{\sum_{m=0}^{k-2} a_{m} a_{m+1}: \sum_{m=0}^{k-1} a_{m}^{2}=1, a_{m} \geq 0(m=0,1, \ldots, k-1)\right\}=\cos \frac{\pi}{k+1} .
$$

3. Estimates of a numerical radius. As is notied in [3], inequality (2) is sharp. However, the following simple example shows that in some cases one can improve inequality (2):

Example. Let $N_{S}=S\left(I-S S^{*}\right)$, where $S, S f=z f$, is a shift operator on the Hardy space $H^{2}=H^{2}(\mathbb{D})$, and let $N$ be a nilpotent operator on a $H$, with power of nilpotency $n, n \geq 3$, and $\|N\|<\frac{1}{2}$. We consider their orthogonal sum:

$$
A=N_{S} \oplus N
$$

It is clear that $N_{S}^{2}=0, A^{n}=0$ and $\|A\|=1$. Since $W\left(N_{S}\right)=\overline{\mathbb{D}}_{1 / 2}$ (see $\left.[12,13]\right)$, $w(N)<\frac{1}{2}$ and $W(A)$ is a convex hull of numerical ranges of addendums, we have $W(A)=\overline{\mathbb{D}}_{1 / 2}$, and therefore $w(A)=\frac{1}{2}<\cos \frac{\pi}{n+1}$. The latter shows that for the numerical radius of the considered nilpotent operator $A$, a stronger estimate is valid.

Note that in the considered example, the numerical radius of the operator $A$ is achieved in the element $\frac{1+z}{\sqrt{2}} \oplus 0$ belonging to $\operatorname{ker}\left(A^{2}\right)$ (to make sure of that, it is sufficient to note that $\left|\left(N_{S} f, f\right)\right|=|\hat{f}(0) \hat{f}(1)| \leq \frac{1}{2}$ for any $\left.f \in\left(H^{2}\right)_{1}\right)$. This suggests an idea that conditions of achievability of the numerical radius of the operator on root subspaces have to play decisive role in obtaining more subtle estimates for a numerical radius of operators. The theorems stated below affirm such point of view.

The following our result can be also considered as an extension of already mentioned Haagerup - de la Harpe's result to some operators from the class $C_{.0}$ that are not nilpotent (recall that the unitary equivalance of operators preserves the numerical radius).

Theorem 1. Let $\Theta$ be a $*$-inner function (i.e., $\Theta(\xi) \Theta(\xi)^{*}=I_{E}$ for a.a. $\xi \in \mathbb{T}$ ), let $\mathcal{K}_{\Theta}=H^{2}(E) \ominus \Theta H^{2}\left(E^{\prime}\right)\left(E\right.$ is some auxillary Hilbert space and $E^{\prime} \subset E$ ) be a model subspace and let $M_{\Theta}=P_{\Theta} z \mid \mathcal{K}_{\Theta}$ be a corresponding model operator. Suppose that for some $n \geq 2$, there exists $x \in \operatorname{ker} M_{\Theta}^{n} \cap\left(\mathcal{K}_{\Theta}\right)_{1}$ such that $w\left(M_{\Theta}\right)=\left|\left(M_{\Theta} x, x\right)\right|$. Then $w\left(M_{\Theta}\right) \leq \cos \frac{\pi}{n+1}$.

Proof. In fact, the assertion $x \in \operatorname{ker} M_{\Theta}^{n}$ is equivalent to the assertion

$$
\Theta^{*} x \in H_{-}^{2}(E) \cap \bar{z}^{n} H^{2}\left(E^{\prime}\right),
$$

that is $\Theta^{*} x=\sum_{k=1}^{n} a_{k} \bar{z}^{k}$, and since $\|x\|=1$ and $\Theta$ is an $*$-inner function,we have $\left\|\Theta^{*} x\right\|=1$, that is $\sum_{k=1}^{n}\left\|a_{k}\right\|^{2}=1$; here, $H_{-}^{2}(E) \stackrel{\text { df }}{=} L^{2}(E) \ominus H^{2}(E)$. Now an elementary argument together with Lemma 1 yields a required estimate: 


$$
\begin{gathered}
w\left(M_{\Theta}\right)=\left|\left(M_{\Theta} x, x\right)\right|=|(z x, x)|= \\
=\left|\left(z \Theta^{*} x, \Theta^{*} x\right)\right|=\left|\sum_{k=1}^{n-1}\left(a_{k+1}, a_{k}\right)\right| \leq \\
\leq \sum_{k=1}^{n-1}\left\|a_{k}\right\|\left\|a_{k+1}\right\| \leq \cos \frac{\pi}{n+1} .
\end{gathered}
$$

The theorem is proved.

In particular, it follows from this theorem that, for a numerical radius of the nilpotent model operator $M_{\Theta}$ with power of nilpotency $n(n \geq 3)$ satisfying the condition of Theorem 1 for some $k, 2 \leq k<n$, there is the estimate

$$
w\left(M_{\Theta}\right) \leq \cos \frac{\pi}{k+1}
$$

which is more subtle than the estimate $w\left(M_{\Theta}\right) \leq \cos \frac{\pi}{n+1}$.

There are some more results on this direction.

Proposition 1. Let $T \in B(H)$ be a contraction such that, for some integer $n>0$, there exists $x \in \operatorname{ker} T^{n} \cap(H)_{1}$ such that $w(T)=|(T x, x)|$. Then $w(T) \leq \cos \frac{\pi}{n+1}$.

Proof. Actually, the assertion of this proposition is a "nonmodel variant" of Theorem 1. Really, for arbitrary $r<1$, the operator $r T$ has two-sided inner characteristic function (because $\|r T\|<1$, and therefore $r T \in C_{00}$ ). Therefore, taking into account that Theorem 1 is also true for the $C_{00}$-class model operators, we obtain that

$$
w(T)=w(r T) \leq \frac{1}{r} \cos \frac{\pi}{n+1} .
$$

It remains to pass to the limit as $r \rightarrow 1$.

Proposition 2. Let $T \in B(H)$ be a contraction such that, for any $\varepsilon>0$, there exists $x_{\varepsilon} \in(H)_{1}$ such that $\left\|T^{n} x_{\varepsilon}\right\|<\varepsilon$ for some $n \geq 2$ and $w(T) \leq\left|\left(T x_{\varepsilon}, x_{\varepsilon}\right)\right|+\varepsilon$. Then $w(T) \leq \cos \frac{\pi}{n+1}$.

Proof. In fact, using the Berberian construction [14], $H \rightarrow H^{0}, T \rightarrow T^{0}(T \in$ $\left.\in B(H), T^{0} \in B\left(H^{0}\right)\right)$, one can reduce the condition of a proposition to the condition of Proposition 1. We recall that the space $H^{0}$ is constructed by the following way:let us take a quatient-space of $l^{\infty}(H)$ of $H$-valued bounded sequences with respect to the linear subspace

$$
N(H) \stackrel{\text { df }}{=}\left\{x=\left(x_{m}\right): \text { L.i.m. }\left(x_{m}, y_{m}\right)=0 \quad \text { for all } \quad y=\left(y_{m}\right) \in l^{\infty}(H)\right\}
$$

with the scalar product

$$
(x+N(x), y+N(y))=\text { L.i.m. }\left(x_{m}, y_{m}\right)
$$

for $x=\left(x_{m}\right), y=\left(y_{m}\right)$, where "L.i.m." is generalized Banach limit on $l^{\infty}$. Then $H^{0}$ is the completion of the last prehilbertian space. Here, $T^{0}$ is defined as

$$
T^{0} x=\left(T x_{n}+N(H)\right)
$$

It is well known (and easily verified) that

ISSN 1027-3190. Укр. мат. журн., 2006, m.58, № 10 


$$
\|T\|=\left\|T^{0}\right\|, \quad \sigma(T)=\sigma\left(T^{0}\right), \quad w(T)=w\left(T^{0}\right) .
$$

Now pass to the reducing the condition of proposition to the condition of Proposition 1 . Choose the sequence of positive numbers $\varepsilon_{m}$ tending to zero and a sequence of vectors $x_{\varepsilon_{m}}$ satisfying the condition of proposition for $\varepsilon=\varepsilon_{m}$ such that $\left|\left(T x_{\varepsilon_{m}}, x_{\varepsilon_{m}}\right)\right| \rightarrow w(T)$. Then for $x=\left(x_{\varepsilon_{m}}\right)$ we have

$$
\begin{gathered}
\left|\left(T^{0} x, x\right)\right|=\mid \text { L.i.m. }\left(T x_{\varepsilon_{m}}, x_{\varepsilon_{m}}\right) \mid= \\
=\left|\lim \left(T x_{\varepsilon_{m}}, x_{\varepsilon_{m}}\right)\right|=\lim \left|\left(T x_{\varepsilon_{m}}, x_{\varepsilon_{m}}\right)\right| \geq w(T)=w\left(T^{0}\right) .
\end{gathered}
$$

Since the inequality $\left|\left(T^{0} x, x\right)\right| \leq w\left(T^{0}\right)$ is always true, it implies the equality $w\left(T^{0}\right)=$ $=\left|\left(T^{0} x, x\right)\right|$. Moreover,

$$
\begin{gathered}
\left(\left(T^{n}\right)^{0} x,\left(T^{n}\right)^{0} x\right)=\text { L.i.m. }\left(T^{n} x_{\varepsilon_{m}}, T^{n} x_{\varepsilon_{m}}\right)= \\
\quad=\text { L.i.m. }\left\|T^{n} x_{\varepsilon_{m}}\right\|^{2} \leq \text { L.i.m. } \varepsilon_{m}^{2}=0
\end{gathered}
$$

that is, $\left(T^{n}\right)^{0} x=0$, whence subject to $\left(T^{n}\right)^{0}=\left(T^{0}\right)^{n}$ we get $\left(T^{0}\right)^{n} x=0$. Thus, $\left(T^{0}\right)^{n} x=0$ and $w\left(T^{0}\right)=\left|\left(T^{0} x, x\right)\right|$. Considering equality (3) and Proposition 1 , we get the desired inequality

$$
w(T) \leq \cos \frac{\pi}{n+1} .
$$

Proposition 3. Let $\Theta_{1}, \Theta_{2}$ be an inner functions. We consider the following operator:

$$
K_{\Theta_{1}, \Theta_{2}} \stackrel{\text { df }}{=}\left[T_{\bar{\Theta}_{1}}, T_{\bar{\Theta}_{2}}\right] \Theta_{1}\left(M_{\Theta_{2}}\right),
$$

where $\left[T_{\bar{\Theta}_{1}}, T_{\Theta_{2}}\right]=T_{\bar{\Theta}_{1}} T_{\Theta_{2}}-T_{\Theta_{2}} T_{\bar{\Theta}_{1}}$ is the commutator of antianalytic Toeplitz operator $T_{\bar{\Theta}_{1}}$ and analytic Toeplitz operator $T_{\Theta_{2}}$, and $\Theta_{1}\left(M_{\Theta_{2}}\right)=P_{\Theta_{2}} \Theta_{1} \mid K_{\Theta_{2}}$ is the function of model operator $M_{\Theta}$. Then $w\left(K_{\Theta_{1}, \Theta_{2}}\right) \leq \frac{1}{2}$.

Proof. The simple calculations show that the operator $K_{\Theta_{1}, \Theta_{2}}$ is the projection of the operator $T_{\bar{\Theta}_{1}} N_{\Theta_{2}} T_{\Theta_{1}}$ on a subspace $\mathcal{K}_{\Theta_{2}}=H^{2} \ominus \Theta H^{2}$, i.e.,

$$
K_{\Theta_{1}, \Theta_{2}}=P_{\Theta_{2}}\left(T_{\bar{\Theta}_{1}} N_{\Theta_{2}} T_{\Theta_{1}}\right) \mid \mathcal{K}_{\Theta_{2}},
$$

where $N_{\Theta_{2}} \stackrel{\text { df }}{=} T_{\Theta_{2}} P_{\Theta_{2}}=T_{\Theta_{2}}\left(I-T_{\Theta_{2}} T_{\bar{\Theta}_{2}}\right)$. In fact, considering that $\left|\Theta_{2}(\xi)\right|=1$ a.a. $\xi \in \mathbb{T}$ (i.e., $T_{\Theta_{2}}$ is an isometry ), for each $f \in \mathcal{K}_{\Theta_{2}}$ we have

$$
\begin{gathered}
P_{\Theta_{2}}\left(T_{\bar{\Theta}_{1}} N_{\Theta_{2}} T_{\Theta_{1}}\right) f=P_{\Theta_{2}} T_{\bar{\Theta}_{1}} T_{\Theta_{2}} P_{\Theta_{2}} \Theta_{1} f= \\
=\left(I-T_{\Theta_{2}} T_{\bar{\Theta}_{2}}\right) T_{\bar{\Theta}_{1}} T_{\Theta_{2}} \Theta_{1}\left(M_{\Theta_{2}}\right) f= \\
=\left(T_{\bar{\Theta}_{1}} T_{\Theta_{2}}-T_{\Theta_{2}} T_{\bar{\Theta}_{2}} T_{\bar{\Theta}_{1}} T_{\Theta_{2}}\right) \Theta_{1}\left(M_{\Theta_{2}}\right) f= \\
=\left(T_{\bar{\Theta}_{1}} T_{\Theta_{2}}-T_{\Theta_{2}} T_{\bar{\Theta}_{2} \Theta_{1}} \Theta_{2}\right) \Theta_{1}\left(M_{\Theta_{2}}\right) f= \\
=\left(T_{\bar{\Theta}_{1}} T_{\Theta_{2}}-T_{\Theta_{2}} T_{\bar{\Theta}_{1}}\right) \Theta_{1}\left(M_{\Theta_{2}}\right) f= \\
=\left[T_{\bar{\Theta}_{1}}, T_{\Theta_{2}}\right] \Theta_{1}\left(M_{\Theta_{2}}\right) f=K_{\Theta_{1}, \Theta_{2}} f .
\end{gathered}
$$

Then for any $x \in\left(\mathcal{K}_{\Theta_{2}}\right)_{1}$ we have 


$$
\left(K_{\Theta_{1}, \Theta_{2}} x, x\right)=\left(P_{\Theta_{2}}\left(T_{\bar{\Theta}_{1}} N_{\Theta_{2}} T_{\Theta_{1}}\right) x, x\right)=\left(N_{\Theta_{2}} \Theta_{1} x, \Theta_{1} x\right) .
$$

Taking into account that $\Theta_{1} x \in\left(H^{2}\right)_{1}, N_{\Theta_{2}}^{2}=0$, and $\left\|N_{\Theta_{2}}\right\|=1$, we get the following relation from the last equality and inequality (2):

$$
w\left(K_{\Theta_{1}, \Theta_{2}}\right) \leq \cos \frac{\pi}{3}=\frac{1}{2} .
$$

The proof is completed.

I am grateful to Yu. V. Turowski for useful discussions.

1. Halmos P. R. A Hilbert space problem book. - New York: Springer, 1982.

2. Gustafson K. E., Rao D. K. M. Numerical range. The field of values of linear operators and matrices. New York: Springer, 1997.

3. Haagerup U., de la Harpe P. The numerical radius of a nilpotent operator on a Hilbert space // Proc. Amer. Math. Soc. - 1992. - 115. - P. 371-379.

4. Pop C. On a result of Haagerup and de la Harpe // Rev. roum. math. pures et appl. - 1998. - 93. - P. 9-10.

5. Badea C, Cassier G. Constrained von Neumann inequalities // Adv. Math. - 2002. - 166. - P. 260-297.

6. Suen C. Y. $\mathrm{W}_{A}$ contactions // Positivity. - 1998. - 2. - P. 301-310.

7. Karaev M. T. The numerical range of a nilpotent operator on a Hilbert space // Proc. Amer. Math. Soc. 2004. - 132, № 8. - P. $2321-2326$.

8. Karaev M. T. New proofs of de la Haagerup-Harpe's inequality // Mat. Zametki. - 2004. - 75, № 5. P. $787-788$.

9. Szökefalvi-Nagy B., Foias C. Harmonic analysis of operators on Hilbert space. - Amsterdam: NorthHolland, 1970.

10. Nikolski N. K. Treatise on the shift operator. - New York: Springer, 1986.

11. Polya G., Szego G. Problems and theorems in analysis. - Berlin: Springer, 1976. - Vol 2.

12. Karaev M. T. On numerical characteristics of some operators associated with isometries // Spectral Theory Operators and Appl. - 1997. - 11. - P. 90-98.

13. Tso S-H., Wu P. Y. Matrical ranges of quadratic operators // Rocky Mountain J. Math. - 1999. - 29. P. $1139-1152$.

14. Berberian S. K. Approximate proper vectors // Proc. Amer. Math. Soc. - 1962. - 13. - P. $111-114$.

Received 05.11.2003, after revision - 03.05.2006 